\title{
Contribution of inherited thrombophilia to recurrent miscarriage in the Polish population
}

\author{
Hubert Wolski ${ }^{1,2}$, Magdalena Barlik ${ }^{1,3}$, Krzysztof Drews ${ }^{1,3}$, Andrzej Klejewski ${ }^{4,5}$, \\ Grażyna Kurzawińska ${ }^{3}$, Marcin Ożarowski ${ }^{6}$, Zdzisław Łowicki ${ }^{6}$, \\ Agnieszka Seremak-Mrozikiewicz'1,3,6 \\ ${ }^{1}$ Division of Perinatology and Women's Diseases, Poznan University of Medical Sciences, Poznan, Poland \\ ${ }^{2}$ Division of Gynecology and Obstetrics, Podhale Multidisciplinary Hospital, Nowy Targ, Poland \\ ${ }^{3}$ Laboratory of Molecular Biology, Division of Perinatology and Women's Diseases, \\ Poznan University of Medical Sciences, Poznan, Poland \\ ${ }^{4}$ Department of Nursing, Poznan University of Medical Sciences, Poznan, Poland \\ ${ }^{5}$ Department of Obstetrics and Women's Diseases, Poznan University of Medical Sciences, Poznan, Poland \\ ${ }^{6}$ Department of Pharmacology and Phytochemistry, Institute of Natural Fibers and Medicinal Plants, Poznan, Poland
}

\begin{abstract}
Introduction: The aim of the study was to evaluate the contribution of genetic variants determining inherited thrombophilia to recurrent miscarriage (RM) in the Polish population. The following polymorphisms were analyzed: $1691 G>A$, $1328 T>C$ of coagulation factor V, 20210G $>A$ of coagulation factor II, R353Q (11496G $>A$ ) of coagulation factor VII, 667C $>T$, $1298 A>C, 1793 G>A$ of MTHFR.
\end{abstract}

Material and methods: A total of 359 women with $\geq 2$ subsequent recurrent miscarriages $(303<13$ weeks of gestation (w.g.) and 56 between 13-22 w.g.) and 400 healthy controls were included in the study. Frequency of the genetic polymorphisms was determined with the PCR/RFLP method.

Results: Higher frequency of the 20210GA genotype was found in the RM < 13 w.g. (2.97 vs. $1.50 \%$ in controls, OR=2.01, ns) and the RM 13-22 w.g. (5.36 vs. $1.50 \%$ in controls, $O R=3.72, p=0.09$ ) subgroups. Statistically significantly higher frequency of the $11496 \mathrm{GA}$ genotype was noted in controls as compared to the RM 13-22 w.g. subgroup (10.71 vs. $23.00 \%$ in controls, $\mathrm{OR}=0.40, \mathrm{p}=0.02$ ). Statistically significantly higher frequency of the $1793 \mathrm{GA}$ genotype was observed in the RM $<13 \mathrm{w} . \mathrm{g}$ subgroup as compared to controls ( 12.21 vs. $7.75 \%$ in controls, $O R=1.66, p=0.03$ ). No significant correlations were found as far as the rest of the analyzed polymorphisms are concerned.

Conclusions: The obtained results suggest that the 1793G>AMTHFR, R353Q(11496G>A) factor VII gene and the 20210G $>A$ factor II gene polymorphisms play a role in the etiology of RM in the Polish population.

Key words: recurrent miscarriage, inherited thrombophilia, genetic polymorphism

Ginekologia Polska 2017; 88, 7: 385-392

\section{INTRODUCTION}

Polymorphic variants of genes involved in the coagulation cascade and fibrinolysis are believed to play a key role in the etiology of recurrent miscarriage (RM). Inherited thrombophilia may result in significant changes in the utero-placental circulation, including placental infarction, atherosclerotic changes in vessels, and placental insufficiency [1-4].
The most common causes of inherited thrombophilia are polymorphisms in genes encoding factor $V$, prothrombin (factor II), factor VII, methylenetetrahydrofolate reductase (MTHFR), and plasminogen activator inhibitor, while protein C, protein S and antithrombin deficiency is less common. Studies confirming a correlation between inherited thrombophilia and recurrent pregnancy loss have been performed all over the world and concerned diverse ethnic populations [5-7]. 
One of a number of hypotheses concerning a correlation between inherited thrombophilia and RM is based on the fact that coagulation cascade stimulation caused by endothelial dysfunction is more likely to occur in women with genetic defects of hemostatic factors predisposing to thrombotic changes in the placental circulation $[8,9]$. The subsequent complications, especially miscarriage, are probably conditioned by impaired placental development and improper placental perfusion. Thrombotic tendency may be manifested by the so-called 'thrombotic damage of placental circulation'. Those changes are known as the 'pregnancy vascular complications' $[10,11]$.

Pregnancy itself is a condition which favors the appearance of physiological hypercoagulability and, with the additional presence of genetic defects of coagulation cascade and fibrinolysis, which may result in serious obstetrical complications, including RM $[12,13]$.

The fact that pregnancy-related complications develop only in some carriers of thrombotic mutations remains a source of much controversy, although environmental factors have been mentioned as the possible cause. Numerous maternal hemostatic proteins cooperate with trophoblastic coagulation cascade components. That process is essential for proper embryogenesis. Coagulation proteins are believed to act also as regulatory and signal factors in immunological reactions and cellular proliferation, although further studies are necessary to fully elucidate the matter $[14,15]$.

\section{OBJECTIVES}

The goal of the research was to evaluate the contribution of genetic variants determining inherited thrombophilia to recurrent miscarriage (RM) in the Polish population. The following polymorphisms were analyzed: $1691 \mathrm{G}>A$, $1328 T>C$ of coagulation factor $V, 20210 G>A$ of coagulation factor II, $R 353 Q(11496 \mathrm{G}>A)$ of coagulation factor VII, 667C $>T$, $1793 G>A$ and $1298 A>C$ of $M T H F R$.

\section{MATERIAL AND METHODS}

A total of 359 women with $\geq 2$ RM and 400 healthy controls were included in the study. Miscarriage was defined as the'loss of pregnancy before 22 completed weeks of gestation (w.g.)'. Gestational age at the time of miscarriage was calculated according to the date of the last menstruation and ultrasound evaluation. All subjects were Caucasian and of Polish origin nationality. The patients were enrolled at the Division of Perinatology and Women's Diseases, Poznan University of Medical Sciences. The study was performed between 2009 and 2015. Local Ethics Committee approved of the study $(1082 / 07,867 / 15,210 / 16)$. All patients gave their written informed consent. All participants were taking $400 \mathrm{mg}$ folic acid per day at the time of the study.

\section{Study group}

The study group was divided into two subgroups: 303 women with $\geq 2$ subsequent $\mathrm{RM}<13$ w.g. during one relationship and 56 women with $\geq 2$ subsequent RM between 13-22 w.g. during one relationship. The presence of protein $C$, protein $S$, antithrombin deficiency as well as antiphospholipid syndrome were excluded in all subjects. Each patient had a negative history of thrombotic events. Patients with known reasons for RM (e.g. anatomical anomalies of the genitourinary tract, chromosomal aberrations, acquired thrombophilia, chronic diseases, infections, hormonal disturbances), cervical insufficiency, or other obstetric complications which could be a cause of RM (e.g. hypertension diagnosed in the course of pregnancy, gestational diabetes mellitus, anatomical and genetic fetal defects, serological conflict) were excluded. Only patients with RM of an unknown origin were included into the analysis.

\section{Control group}

The control group included 400 healthy women with a medical history of at least two uncomplicated pregnancies ended at term with a delivery of a healthy infant. No miscarriages were recorded in this group. All women positive for miscarriage, other obstetric complications caused by thrombotic changes chronic diseases, acquired thrombophilia (antiphospholipid syndrome), and positive history of thrombotic events were excluded from the analysis. Gestational age at the time of delivery was calculated according to the date of the last menstruation and ultrasound evaluation.

Clinical characteristics of the study population are presented in Table 1.

\section{Genetic analysis}

DNA was isolated from blood leucocytes using QIAamp DNA Blood Mini Kit (QIAGEN Inc., Germany). The frequency of the investigated genetic polymorphisms was assessed by polymerase chain reaction (PCR) and restriction fragment length polymorphism (RFLP). Genetic analysis was performed at the Laboratory of Molecular Biology, Division of Perinatology and Women's Diseases, PUMS. Table 2 presents used primers. Investigated polymorphisms were recognized by adequate restriction enzyme hydrolysis. Hydrolyzed PCR products were analyzed on a $2 \%$ agarose gel. Visualization was performed under UV light.

\section{Statistical methods}

Statistical analyses were performed using SPSS22.0 PL for Windows. The $p$-value of $<0.05$ was considered as statistically significant. Genotype frequencies were compared by chi-square test (one-sided Fisher test). The expected genotype frequencies were calculated from allele frequencies with the use of the Hardy-Weinberg equation. 


\section{RESULTS}

There were no statistically significant correlations for the $1691 \mathrm{G}>\mathrm{A}$ factor $\mathrm{V}$ gene polymorphism in both study subgroups and controls. The frequency of the heterozygous 1691GA genotype was comparable in all analyzed groups (7.59\% in the RM $<13$ w.g. subgroup, $5.36 \%$ in the RM 13-

Table 1. Clinical characteristics of the study and control groups.

\begin{tabular}{|c|c|c|c|}
\hline & \multicolumn{2}{|c|}{$\mathrm{RM}(\mathrm{n}=359)$} & \multirow{2}{*}{$\begin{array}{l}\text { Controls } \\
(n=400)\end{array}$} \\
\hline & & $p$ & \\
\hline 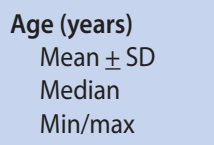 & $\begin{array}{c}30.99 \pm 4.50 \\
31.00 \\
20.00-45.00\end{array}$ & 0.001 & $\begin{array}{c}30.05 \pm 3.81 \\
30.00 \\
22.00-44.00\end{array}$ \\
\hline $\begin{array}{l}\text { Systolic [mm Hg] } \\
\text { Mean } \pm \text { SD } \\
\text { Median } \\
\text { Min/max }\end{array}$ & $\begin{array}{c}109.86 \pm 12.63 \\
110.00 \\
80.00-140.00\end{array}$ & 0.08 & $\begin{array}{c}111.36 \pm 10.70 \\
110.00 \\
80.00-150.00\end{array}$ \\
\hline $\begin{array}{l}\text { Diastolic [mm Hg] } \\
\text { Mean } \pm \text { SD } \\
\text { Median } \\
\text { Min } / \text { max }\end{array}$ & $\begin{array}{c}68.05 \pm 10.11 \\
70.00 \\
50.00-100.00\end{array}$ & 0.0005 & $\begin{array}{l}70.51 \pm 9.24 \\
70.00 \\
50.00-95.00\end{array}$ \\
\hline $\begin{array}{l}\text { Height }[\mathrm{cm}] \\
\quad \text { Mean } \pm \text { SD } \\
\text { Median } \\
\text { Min/max }\end{array}$ & $\begin{array}{c}165.89 \pm 5.58 \\
165.00 \\
150.00-179.00\end{array}$ & 0.19 & $\begin{array}{c}166.42 \pm 5.44 \\
166.00 \\
155.00-180.00\end{array}$ \\
\hline $\begin{array}{l}\text { Weight }[\mathrm{kg}] \\
\text { Mean } \pm \text { SD } \\
\text { Median } \\
\text { Min/max }\end{array}$ & $\begin{array}{c}62.46 \pm 9.25 \\
62.00 \\
43.00-92.00\end{array}$ & 0.002 & $\begin{array}{c}59.83 \pm 9.82 \\
58.00 \\
39.00-110.00\end{array}$ \\
\hline $\begin{array}{l}\text { BMI }\left[\mathrm{kg} / \mathrm{m}^{2}\right] \\
\quad \text { Mean } \pm \text { SD } \\
\text { Median } \\
\text { Min } / \max \end{array}$ & $\begin{array}{c}22.64 \pm 3.25 \\
21.97 \\
17.19-36.85\end{array}$ & 0.0001 & $\begin{array}{c}21.58 \pm 3.26 \\
20.72 \\
16.02-38.57\end{array}$ \\
\hline $\begin{array}{l}\text { Number of RM } \\
1 \text { miscarriage } \\
2 \text { RM } \\
3 \text { or more RM }\end{array}$ & $\begin{array}{c}0 \\
282 \\
77\end{array}$ & - & $\begin{array}{l}0 \\
0 \\
0\end{array}$ \\
\hline
\end{tabular}

-22 w.g. subgroup and $5.25 \%$ in controls, ns). Also, the occurrence of the mutated $1691 \mathrm{~A}$ allele was similar in all investigated groups ( $3.80 \%$ in the $\mathrm{RM}<13$ w.g. subgroup, $2.68 \%$ in the RM 13-22 w.g. subgroup, $2.88 \%$ in controls, ns) (Table 3).

The analysis of the $1328 T>C$ factor $V$ gene polymorphism revealed comparable frequency of its variants. Congenial occurrence of heterozygous 1328TC and homozygous $1328 \mathrm{CC}$ genotypes was observed in the $\mathrm{RM}<13$ w.g. subgroup (23.76\% and 1.65\%), in the RM 13-22 w.g. subgroup (16.07\% and $1.79 \%$ ) and in controls (22.25\% and $1.25 \%)$. As for the mutated $1328 \mathrm{C}$ allele, a similar frequency was also noted: $13.53 \%$ in the RM < 13 w.g. subgroup, $9.82 \%$ in the RM 13-22 w.g. subgroup, and $12.38 \%$ in controls (Table 4).

While analyzing the $20210 \mathrm{G}>A$ factor II gene polymorphism, higher frequency of the heterozygous 20210GA genotype was observed in the $\mathrm{RM}<13$ w.g. subgroup ( 2.97 vs. $1.50 \%$ in controls, $O R=2.01, \mathrm{~ns}$ ) and in the $\mathrm{RM}$ $13-22$ w.g. subgroup (5.36 vs. $1.50 \%$ in controls, $O R=3.72$, $p=0.09)$. The same correlation was found in the mutated $20210 \mathrm{~A}$ allele - its frequency was higher in the RM $<13 \mathrm{RM}$ subgroup ( 1.49 vs. $0.75 \%$ in controls, $\mathrm{OR}=1.99, \mathrm{~ns}$ ) and in the RM $13-22$ w.g. subgroup ( 2.68 vs. $0.75 \%$ in controls, $W R=3.72, p=0.09$ ) (Table 5).

Similar observations were made for the $R 353 Q$ (11496G>A) factor VII gene polymorphism. Statistically significantly higher frequency of the heterozygous $11496 \mathrm{GA}$ genotype was noted in controls as compared to the RM $13-22$ w.g. subgroup ( 10.71 vs. $23.00 \%$ in controls, $O R=0.40$, $\mathrm{p}=0.02)$. Comparable statistical differences concerned genotypes containing the mutated $11496 \mathrm{~A}$ allele $(G A+A A)$ in the $\mathrm{RM}<13$ w.g. subgroup and in controls ( $12.50 \mathrm{vs.} 24.50 \%$, $\mathrm{OR}=0.44, \mathrm{p}=0.029$ ). The frequency of the mutated $11496 \mathrm{~A}$ allele was also higher in controls as compared to the RM $13-22$ w.g. subgroup (7.14 vs. $13.00 \%$ in controls, $\mathrm{OR}=0.51$, $\mathrm{p}=0.05$ ) (Table 6).

Table 2. Primers used in genetic analysis

\begin{tabular}{|c|c|c|c|}
\hline Gene & Polymorphism & Primer sequence & References \\
\hline \multirow{3}{*}{ MTHFR } & $\begin{array}{l}677 \mathrm{C}>T \\
(A 222 V)\end{array}$ & $\begin{array}{l}\text { 5'TGA AGG AGA AGG TGT CTG CGG GA 3' } \\
\text { 5'AGG ACG GTG CGG TGA GAG TG 3' }\end{array}$ & Frost et al. 1995 \\
\hline & $\begin{array}{l}1298 A>C \\
(E 429 A)\end{array}$ & $\begin{array}{c}5^{\prime} \text { CTT CTA CCT GAA GAG CAA GTC-3' } \\
5^{\prime} \text { CAT GTC CAC AGC ATG GAG-3' }\end{array}$ & Hanson et al. 2001 \\
\hline & $\begin{array}{l}1793 G>A \\
\text { (R594Q) }\end{array}$ & $\begin{array}{l}\text { 5' CTC TGT GTG TGT GTG CAT GTG TGC G 3' } \\
\text { 5' GGG ACA GGA GTG GCT CCA ACG CAG G 3' }\end{array}$ & Rady et al. 2002 \\
\hline \multirow{2}{*}{ FV } & $\begin{array}{l}1691 G>A \\
(R 506 Q)\end{array}$ & $\begin{array}{l}5^{\prime} \text { TGC CCA GTG CTT AAC AAG ACC A 3' } \\
5^{\prime} \text { CTT GAA GGA AAT GCC CCA TTA } 3^{\prime}\end{array}$ & Bertina et al. 1994 \\
\hline & $\begin{array}{l}1328 T>C \\
(M 385 T)\end{array}$ & $\begin{array}{l}\text { 5'ACA TAC AGT GAA TCC CAG TA } 3^{\prime} \\
\text { 5'ATG AGC ATC TTT TTC TTT TA3' }\end{array}$ & Faisel et al. 2004 \\
\hline FII & $20210 G>A$ & $\begin{array}{l}\text { 5'TCT AGA AAC AGT TGC CTG GC 3' } \\
\text { 5' ATA GCA CTG GGA GCA TTG AAG C3' }\end{array}$ & Poort et al. 1996 \\
\hline FVII & $\begin{array}{l}10916 G>A \\
(R 353 Q)\end{array}$ & $\begin{array}{l}\text { 5' GGG AGA CTC CCC AAA TAT CAC 3' } \\
5^{\prime} \text { ACG CAG CCT TGG CTT TCT CTC 3' }\end{array}$ & Green et al. 1991 \\
\hline
\end{tabular}


Table 3. The frequency of genotypes and alleles of the $1691 \mathrm{G}>A$ factor $V$ gene polymorphism in the study group and controls

\begin{tabular}{|c|c|c|c|c|c|c|c|c|c|c|}
\hline \multirow{3}{*}{$\begin{array}{l}\text { FV } \\
\text { 1691G>A } \\
\text { Genotypes }\end{array}$} & \multicolumn{8}{|c|}{ Study group RM ( $n=359$ ) } & \multirow{2}{*}{\multicolumn{2}{|c|}{ Control group $(n=400)$}} \\
\hline & \multicolumn{4}{|c|}{$R M<13$ w.g. $(n=303)$} & \multicolumn{4}{|c|}{ RM 13-22 w.g. $(n=56)$} & & \\
\hline & $\begin{array}{l}\text { Observed } \\
\text { value } \\
\text { n (\%) }\end{array}$ & $\begin{array}{c}\text { Expected } \\
\text { value } \\
(\%)\end{array}$ & OR & $\mathbf{p}$ & $\begin{array}{l}\text { Observed } \\
\text { value } \\
\text { n (\%) }\end{array}$ & $\begin{array}{c}\text { Expected } \\
\text { value } \\
(\%)\end{array}$ & OR & $\mathbf{p}$ & $\begin{array}{l}\text { Observed } \\
\text { value } \\
\text { n (\%) }\end{array}$ & $\begin{array}{l}\text { Expected } \\
\text { value } \\
(\%)\end{array}$ \\
\hline 1691GG & $280(92.41)$ & 92.55 & 0.71 & 0.17 & 53 (94.64) & 94.72 & 1.03 & 0.63 & $378(94.50)$ & 94.33 \\
\hline $1691 G A$ & $23(7.59)$ & 7.30 & 1.48 & 0.13 & $3(5.36)$ & 5.21 & 1.02 & 0.58 & $21(5.25)$ & 5.59 \\
\hline 1691AA & $0(0.00)$ & 0.15 & - & - & $0(0.00)$ & 0.07 & - & - & $1(0.25)$ & 0.08 \\
\hline Total & $303(100.00)$ & 100.00 & & & $56(100.00)$ & 100.00 & & & $400(100.00)$ & 100.00 \\
\hline \multicolumn{11}{|l|}{ Alleles } \\
\hline $1691 G$ & $583(96.20)$ & - & 0.75 & 0.21 & $109(97.32)$ & - & 1.08 & 0.60 & $777(97.12)$ & - \\
\hline $1691 A$ & $23(3.80)$ & - & 1.33 & 0.21 & $3(2.68)$ & - & 0.93 & 0.60 & $23(2.88)$ & - \\
\hline Total & $606(100.00)$ & - & & & $112(100.00)$ & - & & & $800(100.00)$ & - \\
\hline
\end{tabular}

Study subgroups were compared to the control group; $\mathrm{p}$ - one-sided exact Fisher test

\begin{tabular}{|c|c|c|c|c|c|c|c|c|c|c|}
\hline \multirow{3}{*}{$\begin{array}{l}\text { FV } \\
\text { 1328T>C } \\
\text { (M385T) } \\
\text { Genotypes }\end{array}$} & \multicolumn{8}{|c|}{ Study group RM ( $n=359$ ) } & \multirow{2}{*}{\multicolumn{2}{|c|}{ Control group $(n=400)$}} \\
\hline & \multicolumn{4}{|c|}{$R M<13$ w.g. $(n=303)$} & \multicolumn{4}{|c|}{ RM 13-22 w.g. $(n=56)$} & & \\
\hline & $\begin{array}{c}\text { Observed } \\
\text { value } \\
\text { n (\%) }\end{array}$ & $\begin{array}{c}\text { Expected } \\
\text { value } \\
(\%)\end{array}$ & OR & $\mathbf{p}$ & $\begin{array}{l}\text { Observed } \\
\text { value } \\
\text { n (\%) }\end{array}$ & $\begin{array}{c}\text { Expected } \\
\text { value } \\
(\%)\end{array}$ & OR & p & $\begin{array}{c}\text { Observed } \\
\text { value } \\
\text { n (\%) }\end{array}$ & $\begin{array}{c}\text { Expected } \\
\text { value } \\
(\%)\end{array}$ \\
\hline $1328 T T$ & $226(74.59)$ & 74.77 & 0.90 & 0.31 & $46(82.14)$ & 81.32 & 1.41 & 0.22 & $306(76.50)$ & 76.78 \\
\hline $1328 T C$ & $72(23.76)$ & 23.40 & 1.09 & 0.35 & $9(16.07)$ & 17.71 & 0.67 & 0.19 & $89(22.25)$ & 21.69 \\
\hline $1328 C C$ & $5(1.65)$ & 1.83 & 1.33 & 0.45 & $1(1.79)$ & 0.97 & 1.44 & 0.54 & $5(1.25)$ & 1.53 \\
\hline Total & $303(100.00)$ & 100.00 & & & $56(100.00)$ & 100.00 & & & $400(100.00)$ & 100.00 \\
\hline \multicolumn{11}{|l|}{ Alleles } \\
\hline $1328 T$ & $524(86.47)$ & - & 0.90 & 0.29 & $101(90.18)$ & - & 1.30 & 0.27 & $701(87.62)$ & - \\
\hline $1328 \mathrm{C}$ & $82(13.53)$ & - & 1.11 & 0.29 & $11(9.82)$ & - & 0.77 & 0.27 & 99 (12.38) & - \\
\hline Total & $606(100.00)$ & - & & & $112(100.00)$ & - & & & $800(100.00)$ & - \\
\hline
\end{tabular}

Study subgroups were compared to the control group; $\mathrm{p}$ - one-sided exact Fisher test

Table 5. The frequency of genotypes and alleles of the $20210 \mathrm{G}>\mathrm{A}$ factor II gene polymorphism in the study group and controls

\begin{tabular}{|c|c|c|c|c|c|c|c|c|c|c|}
\hline \multirow{2}{*}{$\begin{array}{l}\text { FII } \\
20210 G>A\end{array}$} & \multicolumn{8}{|c|}{ Study group RM ( $n=359$ ) } & \multirow{2}{*}{\multicolumn{2}{|c|}{ Control group $(n=400)$}} \\
\hline & \multicolumn{4}{|c|}{$R M<13$ w.g. $(n=303)$} & \multicolumn{4}{|c|}{ RM 13-22 w.g. $(n=56)$} & & \\
\hline Genotypes & $\begin{array}{l}\text { Observed } \\
\text { value } \\
\text { n (\%) }\end{array}$ & $\begin{array}{l}\text { Expected } \\
\text { value } \\
\text { (\%) }\end{array}$ & OR & p & $\begin{array}{l}\text { Observed } \\
\text { value } \\
\text { n (\%) }\end{array}$ & $\begin{array}{l}\text { Expected } \\
\text { value } \\
\text { (\%) }\end{array}$ & OR & p & $\begin{array}{l}\text { Observed } \\
\text { value } \\
\text { n (\%) }\end{array}$ & $\begin{array}{l}\text { Expected } \\
\text { value } \\
(\%)\end{array}$ \\
\hline $20210 G G$ & $294(97.03)$ & 97.05 & 0.50 & 0.14 & $53(94.64)$ & 94.72 & 0.27 & 0.09 & $394(98.50)$ & 98.51 \\
\hline 20210GA & $9(2.97)$ & 2.93 & 2.01 & 0.14 & $3(5.36)$ & 5.21 & 3.72 & 0.09 & $6(1.50)$ & 1.49 \\
\hline $20210 A A$ & $0(0.00)$ & 0.02 & - & - & $0(0.00)$ & 0.07 & - & - & $0(0.00)$ & 0.00 \\
\hline Total & $303(100.00)$ & 100.00 & & & $56(100.00)$ & & & & $400(100.0)$ & 100.00 \\
\hline \multicolumn{11}{|l|}{ Alleles } \\
\hline $20210 G$ & $597(98.51)$ & - & 0.50 & 0.14 & 109 (97.32) & - & 0.27 & 0.09 & $794(99.25)$ & - \\
\hline $20210 A$ & $9(1.49)$ & - & 1.99 & 0.14 & $3(2.68)$ & - & 3.72 & 0.09 & $6(0.75)$ & - \\
\hline Total & $606(100.00)$ & - & & & $112(100.00)$ & - & & & $800(100.00)$ & - \\
\hline
\end{tabular}

Study subgroups were compared to the control group; $\mathrm{p}$ - one-sided exact Fisher test 
Table 6. The frequency of genotypes and alleles of the $11496 \mathrm{G}>\mathrm{A}$ factor VII gene polymorphism in the study group and controls

\begin{tabular}{|c|c|c|c|c|c|c|c|c|c|c|}
\hline \multirow{2}{*}{$\begin{array}{l}\text { FVII } \\
\text { R353Q } \\
(11496 G>A)\end{array}$} & \multicolumn{8}{|c|}{ Study group RM ( $n=359$ ) } & \multirow{2}{*}{\multicolumn{2}{|c|}{ Control group $(n=400)$}} \\
\hline & \multicolumn{4}{|c|}{$R M<13$ w.g. $(n=303)$} & \multicolumn{4}{|c|}{ RM 13-22 w.g. $(n=56)$} & & \\
\hline Genotypes & $\begin{array}{c}\text { Observed } \\
\text { value } \\
\text { n (\%) }\end{array}$ & $\begin{array}{l}\text { Expected } \\
\text { value } \\
(\%)\end{array}$ & OR & $\mathbf{p}$ & $\begin{array}{l}\text { Observed } \\
\text { value } \\
\text { n (\%) }\end{array}$ & $\begin{array}{c}\text { Expected } \\
\text { value } \\
(\%)\end{array}$ & OR & $\mathbf{p}$ & $\begin{array}{c}\text { Observed } \\
\text { value } \\
\text { n (\%) }\end{array}$ & $\begin{array}{c}\text { Expected } \\
\text { value } \\
(\%)\end{array}$ \\
\hline $11496 G G$ & $234(77.23)$ & 78.23 & 1.10 & 0.33 & $49(87.50)$ & 86.22 & 2.27 & 0.03 & $302(75.50)$ & 75.69 \\
\hline $11496 \mathrm{GA}$ & $68(22.44)$ & 20.43 & 0.97 & 0.47 & $6(10.71)$ & 13.27 & 0.40 & 0.02 & $92(23.00)$ & 22.62 \\
\hline $11496 A A$ & $1(0.33)$ & 1.34 & 0.22 & 0.12 & $1(1.79)$ & 0.51 & 1.19 & 0.60 & $6(1.50)$ & 1.69 \\
\hline Total & $303(100.00)$ & 100.00 & & & $56(100.00)$ & 100.00 & & & $400(100.00)$ & 100.00 \\
\hline \multicolumn{11}{|l|}{ Alleles } \\
\hline $11496 G$ & $536(88.45)$ & - & 1.14 & 0.23 & $104(92.86)$ & - & 1.94 & 0.05 & $696(87.00)$ & - \\
\hline $11496 A$ & $70(11.55)$ & - & 0.87 & 0.23 & $8(7.14)$ & - & 0.51 & 0.05 & $104(13.00)$ & - \\
\hline Total & $606(100.00)$ & - & & & $112(100.00)$ & - & & & $800(100.00)$ & - \\
\hline
\end{tabular}

Study subgroups were compared to the control group; $\mathrm{p}$ - one-sided exact Fisher test

\begin{tabular}{|c|c|c|c|c|c|c|c|c|c|c|}
\hline \multirow{3}{*}{$\begin{array}{l}\text { MTHFR } \\
677 C>T\end{array}$} & \multicolumn{8}{|c|}{ Study group RM ( $\mathrm{n}=359$ ) } & \multirow{2}{*}{\multicolumn{2}{|c|}{ Control group $(n=400)$}} \\
\hline & \multicolumn{4}{|c|}{$R M<13$ w.g. $(n=303)$} & \multicolumn{4}{|c|}{ RM 13-22 w.g. $(n=56)$} & & \\
\hline & $\begin{array}{c}\text { Observed } \\
\text { value } \\
\text { n (\%) }\end{array}$ & $\begin{array}{l}\text { Expected } \\
\text { value } \\
(\%)\end{array}$ & OR & $\mathbf{p}$ & $\begin{array}{c}\text { Observed } \\
\text { value } \\
\text { n (\%) }\end{array}$ & $\begin{array}{c}\text { Expected } \\
\text { value } \\
(\%)\end{array}$ & OR & $\mathbf{p}$ & $\begin{array}{c}\text { Observed } \\
\text { value } \\
\text { n (\%) }\end{array}$ & $\begin{array}{c}\text { Expected } \\
\text { value } \\
(\%)\end{array}$ \\
\hline $677 C C$ & $139(45.88)$ & 44.89 & 0.84 & 0.14 & $26(46.43)$ & 47.26 & 0.86 & 0.35 & $201(50.25)$ & 50.05 \\
\hline $677 C T$ & $128(42.24)$ & 44.22 & 1.05 & 0.40 & $25(44.64)$ & 42.97 & 1.16 & 0.35 & $164(41.00)$ & 41.39 \\
\hline $677 T T$ & $36(11.88)$ & 10.89 & 1.41 & 0.11 & $5(8.93)$ & 9.77 & 1.02 & 0.56 & $35(8.75)$ & 8.56 \\
\hline Total & $303(100.00)$ & 100.00 & & & $56(100.00)$ & 100.00 & & & $400(100.00)$ & 100.00 \\
\hline \multicolumn{11}{|l|}{ Alleles } \\
\hline $677 C$ & $406(67.00)$ & - & 0.84 & 0.07 & $77(68.75)$ & - & 0.91 & 0.37 & $566(70.75)$ & - \\
\hline $677 T$ & $200(33.00)$ & - & 1.19 & 0.07 & $35(31.25)$ & - & 1.10 & 0.37 & $234(29.25)$ & - \\
\hline Total & $606(100.00)$ & - & & & $112(100.00)$ & - & & & $800(100.00)$ & - \\
\hline
\end{tabular}

Study subgroups were compared to the control group; $\mathrm{p}$ - one-sided exact Fisher test

As for the $677 C>$ TMTHFR gene polymorphism, the occurrence of genotypes and alleles was similar in all analyzed groups. The frequency of the heterozygous 677CT genotype was comparable in both study subgroups and in controls (42.24\% in the RM < 13 w.g. subgroup, $44.64 \%$ in the RM 13-22 w.g. subgroup, $41.00 \%$ in controls, ns). In case of the mutated 677TT genotype, its frequency was $11.88 \%$ in the $\mathrm{RM}<13$ w.g. subgroup, 8.93\% in the RM 13-22 w.g. subgroup, and $8.75 \%$ in controls, ns. A similar correlation was found for allele frequency (Table 7).

For the 1298A>CMTHFR gene polymorphism, the following genotype distribution was observed: in the $\mathrm{RM}<13$ w.g. subgroup - 1298AA: 1298AC: $1298 C C=40.59: 47.20$ : $12.21 \%$, in the RM 13-22 w.g. subgroup - 1298AA: 1298AC: $1298 C C=51.79: 35.71: 12,50 \%$, in controls $-1298 A A$ : $1298 A C: 1298 C C=44.75: 43.00: 12.25 \%$. No statistically sig- nificant correlations were found. The occurrence of the alleles was as follows: in the $\mathrm{RM}<13$ w.g. subgroup - 1298A: $1298 \mathrm{C}=64.19: 35.81 \%$, in the RM 13-22 w.g. subgroup - 1298A: $1298 \mathrm{C}=69.64: 30.36 \%$ in controls $-1298 \mathrm{~A}$ : $1298 \mathrm{C}=66.25: 33.75 \%$ (Table 8).

A similar finding was made about the $17936>A$ MTH$F R$ gene polymorphism. Statistically significantly higher frequency of the heterozygous 1793GA genotype in the $\mathrm{RM}<13$ w.g. subgroup was noted (12.21 vs. $7.75 \%$ in controls, $\mathrm{OR}=1.66, \mathrm{p}=0.03)$. Also, the occurrence of genotypes containing the mutated $1793 A$ allele $(1793 G A+1793 A A)$ was significantly higher in the $\mathrm{RM}<13$ w.g. subgroup ( 12.54 vs. $8.00 \%$ in controls, $O R=1.65, p=0.03$ ). A statistically significant correlation was found also for the frequency of the mutated $1793 \mathrm{~A}$ allele, which was higher in the $\mathrm{RM}<13$ w.g. subgroup ( 6.44 vs. $4.13 \%$ in controls, $\mathrm{OR}=1.60$, 
Table 8. The frequency of genotypes and alleles of the 1298A>C MTHFR gene polymorphism in in the study group and controls

\begin{tabular}{|c|c|c|c|c|c|c|c|c|c|c|}
\hline \multirow{3}{*}{$\begin{array}{l}\text { MTHFR } \\
1298 A>C \\
\text { Genotypes }\end{array}$} & \multicolumn{8}{|c|}{ Study group RM ( $=359$ ) } & \multirow{2}{*}{\multicolumn{2}{|c|}{ Control group $(n=400)$}} \\
\hline & \multicolumn{4}{|c|}{$R M<13$ w.g. $(n=303)$} & \multicolumn{4}{|c|}{ RM 13-22 w.g. $(n=56)$} & & \\
\hline & $\begin{array}{l}\text { Observed } \\
\text { value } \\
\text { n (\%) }\end{array}$ & $\begin{array}{l}\text { Expected } \\
\text { value } \\
(\%)\end{array}$ & OR & $\mathbf{p}$ & $\begin{array}{l}\text { Observed } \\
\text { value } \\
\text { n (\%) }\end{array}$ & $\begin{array}{l}\text { Expected } \\
\text { value } \\
(\%)\end{array}$ & OR & $\mathbf{p}$ & $\begin{array}{l}\text { Observed } \\
\text { value } \\
\text { n (\%) }\end{array}$ & $\begin{array}{c}\text { Expected } \\
\text { value } \\
(\%)\end{array}$ \\
\hline $1298 A A$ & $123(40.59)$ & 41.21 & 0.84 & 0.15 & $29(51.79)$ & 48.50 & 1.33 & 0.20 & $179(44.75)$ & 43.89 \\
\hline $1298 A C$ & $143(47.20)$ & 45.97 & 1.18 & 0.15 & $20(35.71)$ & 42.28 & 0.74 & 0.19 & $172(43.00)$ & 44.72 \\
\hline $1298 C C$ & $37(12.21)$ & 12.82 & 0.99 & 0.54 & $7(12.50)$ & 9.22 & 1.02 & 0.55 & $49(12.25)$ & 11.39 \\
\hline Total & $303(100.00)$ & 100.00 & & & $56(100.00)$ & 100.00 & & & $400(100.00)$ & 100.00 \\
\hline \multicolumn{11}{|l|}{ Alleles } \\
\hline $1298 A$ & $389(64.19)$ & - & 0.91 & 0.23 & 78 (69.64) & - & 1.17 & 0.27 & $530(66.25)$ & - \\
\hline $1298 C$ & $217(35.81)$ & - & 1.10 & 0.23 & $34(30.36)$ & - & 0.86 & 0.27 & $270(33.75)$ & - \\
\hline Total & $606(100.00)$ & - & & & $112(100.00)$ & - & & & $800(100.00)$ & - \\
\hline
\end{tabular}

Study subgroups were compared to the control group; $\mathrm{p}$ - one-sided exact Fisher test

Table 9. The frequency of genotypes and alleles of the 1793G>A MTHFR gene polymorphism in the study group and controls

\begin{tabular}{|c|c|c|c|c|c|c|c|c|c|c|}
\hline \multirow{3}{*}{$\begin{array}{l}\text { MTHFR } \\
\text { 1793G>A } \\
\text { Genotypes }\end{array}$} & \multicolumn{8}{|c|}{ Study group RM ( $n=359)$} & \multirow{2}{*}{\multicolumn{2}{|c|}{ Control group $(n=400)$}} \\
\hline & \multicolumn{4}{|c|}{$R M<13$ w.g. $(n=303)$} & \multicolumn{4}{|c|}{ RM 13-22 w.g. $(n=56)$} & & \\
\hline & $\begin{array}{l}\text { Observed } \\
\text { value } \\
\text { n (\%) }\end{array}$ & $\begin{array}{l}\text { Expected } \\
\text { value } \\
\text { (\%) }\end{array}$ & OR & p & $\begin{array}{l}\text { Observed } \\
\text { value } \\
\text { n (\%) }\end{array}$ & $\begin{array}{l}\text { Expected } \\
\text { value } \\
\text { (\%) }\end{array}$ & OR & $\mathbf{p}$ & $\begin{array}{l}\text { Observed } \\
\text { value } \\
\text { n (\%) }\end{array}$ & $\begin{array}{l}\text { Expected } \\
\text { value } \\
(\%)\end{array}$ \\
\hline $1793 G G$ & $265(87.46)$ & 87.54 & 0.61 & 0.03 & $50(89.29)$ & 89.57 & 0.72 & 0.32 & $368(92.00)$ & 91.92 \\
\hline $1793 G A$ & $37(12.21)$ & 12.04 & 1.66 & 0.03 & $6(10.71)$ & 10.14 & 1.43 & 0.29 & $31(7.75)$ & 7.91 \\
\hline $1793 A A$ & $1(0.33)$ & 0.42 & 1.32 & 0.68 & $0(0.00)$ & 0.29 & - & - & $1(0.25)$ & 0.17 \\
\hline Total & $303(100.00)$ & 100.00 & & & $56(100.00)$ & 100.00 & & & $400(100.00)$ & 100.00 \\
\hline \multicolumn{11}{|l|}{ Alleles } \\
\hline $1793 G$ & $567(93.56)$ & - & 0.63 & 0.03 & $106(94.64)$ & - & 0.76 & 0.34 & $767(95.87)$ & - \\
\hline $1793 A$ & $39(6.44)$ & - & 1.60 & 0.03 & $6(5.36)$ & - & 1.32 & 0.34 & $33(4.13)$ & - \\
\hline Total & $606(100.00)$ & - & & & $112(100.00)$ & - & & & $800(100.00)$ & - \\
\hline
\end{tabular}

Study subgroups were compared to the control group; $\mathrm{p}$ - one-sided exact Fisher test

$\mathrm{p}=0.03)$, whereas there were no significant dissimilarities in the RM 13-22 w.g. subgroup and controls (Table 9).

\section{DISCUSSION}

The present study involved patients with RM as well as healthy controls. It is one of the biggest analyses concerning inherited thrombophilia and RM in the Polish population. The study group is fully representative for that kind of research. Owing to the sample size, the statistical tests are reliable. Significant differences of frequencies of genetic variants between diverse ethnic and regional groups have been reported and that is why eligibility criteria should also include ethnic homogeneity. In our study, all women were Caucasian and of Polish origin. In similar studies, comparing healthy and unhealthy unrelated groups of patients, adequate exclusion and inclusion criteria are of vital importance. Inadequate eligibility criteria and insufficient sample size are the most common reasons of obtaining conflicting results concerning the frequency of genetic polymorphisms [16]. Noteworthy, the abovementioned study considered $7 \mathrm{ge}$ netic polymorphisms in 4 genes of coagulation cascade, folate and homocysteine metabolism.

Factor V Leiden $(1691 \mathrm{G}>A)$ is one of the most commonly analyzed factors involved in the etiology of RM and has been suggested to be the dominant cause of miscarriage in the first and second trimesters of pregnancy. A multi-center study by Skrzypczak et al., performed in 2012, investigated 396 patients with at least one pregnancy loss (122 women with 3 or more early RM, 87 with late RM and 46 with IUFD) and 50 women with a negative history of pregnancy loss. Their results revealed a possible role of the factor $\mathrm{V}$ Leiden in the etiology of RM. Additionally, those authors emphasized the need to perform factor $V$ Leiden screening tests among patients with the diagnosis of RM [17]. A study 
by Sergi et al., performed in 2015, concerned 9 analyses of the Leiden mutation and its significance in RM in the first trimester of pregnancy. The study involved 2147 patients (1305 with RM and 842 healthy women). Higher frequency of factor $V$ Leiden was observed in the study group. These authors voiced the need to investigate the presence of factor V Leiden in each woman with RM of an unknown origin. Moreover, they recommended antithrombotic prophylaxis in all patients with RM and the presence of factor V Leiden [18]. On the other hand, there are also some studies which do not confirm correlation of factor $V$ Leiden with increased risk for RM, which is consistent with our findings. Bauman et al., performed a multi-center analysis of the frequency of factor V Leiden, prothrombin mutation and 677C > TMTHFR polymorphism in a group of 641 patients ( 240 with 2 RM and 401 with 3 or more RM) and 157 healthy controls. All women were Caucasian. No statistically significant differences between the analyzed groups were found [19].

Relatively few studies have dealt with the 1328T>C factor $V$ gene polymorphism. An interesting analysis was performed in the Polish population. The study group included 136 patients with 2 or more RM and 106 controls. The $1691 \mathrm{G}>A$ and $1328 T>C$ polymorphisms were evaluated in all subjects. Heterozygous $1328 T C$ genotype was more frequent in women with RM before 7 w.g. These authors recommend $1328 T>C$ factor $V$ gene polymorphism screening in women with a positive history of RM [20].

An analysis of the correlations of the $20210 G>A$ prothrombin gene polymorphism has been already performed in the Polish population. In the study of Barlik et al., 20210G $>A$ and 19911A>G prothrombin gene polymorphisms were evaluated in a group of 150 women with 2 or more RM and 180 controls. An overrepresentation of the 20210 GA genotype ( $2.7 \%$ vs. $1.1 \%$ in controls, $O R=2.44, n s)$ and the $20210 \mathrm{~A}$ allele $(1.3 \%$ vs. $0.6 \%$ in controls, $\mathrm{OR}=2.42$, ns) was observed in the RM group. These authors suggested a possible role of the $20210 \mathrm{G}>A$ prothrombin gene polymorphism in the etiology of RM [21]. Special attention should be paid to the meta-analysis of Gao et al., involving 37 studies, including 5400 women with 2 or more RM and 4640 controls. It revealed a correlation of the $20210 G>A$ polymorphism with increased risk for pregnancy loss among the European population and women over 29 years of age [22].

The decision to evaluate the $R 353 Q(11496 G>A)$ factor VII polymorphism in the etiology of RM was inspired by reports from the field of cardiology. Factor VII plasma concentration and activity are potential risk factors for thrombotic changes. In carriers of the mutated Gln353 allele factor VII, plasma concentration and activity are decreased, leading to lower risk of thrombotic events [23]. Moreover, a study by Seremak-Mrozikiewicz et al., including 104 women with RM between 6-13 w.g. and 163 controls, revealed a protec- tive role of the Gln353 allele as far as the occurrence of RM was concerned [24], which is consistent with our findings.

A considerable amount of research has focused on the possible role of $667 C>T, 1298 A>C, 1793 G>A$ polymorphisms of the MTHFR gene in the etiology of RM. One study in the Polish population (104 women with RM and 169 controls) has been already performed. Higher frequency of the heterozygous 1793GA genotype in the RM group was noted $(15.38 \%$ vs. $4.14 \%$ in controls, $O R=4.21, p=0.003)$. There were no statistical differences regarding the $667 C>T$ and 1298 A > C genetic variants [25].

A meta-analysis performed in 2006 by Robertson et al., is a very comprehensive research which involved 25 studies on miscarriage in the early pregnancy, RM in the first trimester of pregnancy, and late abortions. The role of the $1691 \mathrm{G}>A$ factor $V$ gene polymorphism and the 20210G >A factor II gene polymorphism in the etiology of RM in the first trimester and late abortions was identified. Also, much attention was paid to the meaning of acquired hyperhomocysteinemia in the increased risk for RM. Interestingly, a correlation between anticardiolipin antibodies and $\mathrm{RM}$ in the first trimester of pregnancy and late abortions was reported [26].

\section{CONCLUSIONS}

1. A significantly higher frequency of the mutated variants of the $1793 \mathrm{G}>$ A polymorphism of the MTHFR gene in the $\mathrm{RM}<13$ w.g. subgroup indicates the role of this polymorphism in the etiology of early miscarriage.

2. A role of the wild-type $353 R R$ (11496GG) genotype and wild-type $353 R$ (11496G) allele of the $R 353 Q(11496 G>A)$ factor VII gene polymorphism in the etiology of late miscarriages (RM between 13-22 w.g.) was revealed.

3. The observed overrepresentation of the mutated genotype and allele of the 20210G $>A$ factor II gene polymorphism in the entire study group may suggest a possible role of these variants in the etiology of RM.

4. Statistically significant correlations are consistent with reports of other authors, which further emphasizes the value of our findings.

\section{Ethics approval and consent to participate}

All procedures involving human participants were performed in accordance with the ethical standards of the institutional and national research committee and with the 1964 Helsinki declaration and its later amendments. Informed consent was obtained from all individual participants included in the study. Local Ethics Committee approved of the study design.

\section{Conflict of interests}

The authors declare that they have no conflict of interests. 


\section{REFERENCES}

1. Saito $\mathrm{S}$. The causes and treatment of recurrent pregnancy loss. JMAJ. 2009; 52: 97-102.

2. Rambaldi MP, Mecacci F, Guaschino S, et al. Inherited and acquired thrombophilias. Reprod Sci. 2014; 21(2): 167-182, doi: 10.1177/ /1933719113497282, indexed in Pubmed: 23899550.

3. Middeldorp S. Anticoagulation in pregnancy complications. Hematology Am Soc Hematol Educ Program. 2014; 2014(1): 393-399, doi: 10.1182/asheducation-2014.1.393, indexed in Pubmed: 25696884.

4. Ford HB, Schust DJ. Recurrent pregnancy loss: etiology, diagnosis, and therapy. Rev Obstet Gynecol. 2009; 2(2): 76-83, indexed in Pubmed: 19609401

5. Alonso A, Soto I, Urgellés MF, et al. Acquired and inherited thrombophilia in women with unexplained fetal losses. Am J Obstet Gynecol. 2002; 187(5): 1337-1342, indexed in Pubmed: 12439528.

6. Bennett SA, Bagot CN, Arya R. Pregnancy loss and thrombophilia: the elusive link. Br J Haematol. 2012; 157(5): 529-542, doi: 10.1111/j.1365-2141.2012.09112.x, indexed in Pubmed: 22449204.

7. Vaiman D. Genetic regulation of recurrent spontaneous abortion in humans. Biomed J. 2015; 38(1): 11-24, doi: 10.4103/2319-4170.133777, indexed in Pubmed: 25179715.

8. Cao Y, Zhang Z, Xu J, et al. The association of idiopathic recurrent pregnancy loss with polymorphisms in hemostasis-related genes. Gene. 2013; 530(2): 248-252, doi: 10.1016/j.gene.2013.07.080, indexed in Pubmed: 23954867.

9. Carp H, Salomon O, Seidman D, et al. Prevalence of genetic markers for thrombophilia in recurrent pregnancy loss. Hum Reprod. 2002; 17(6): 1633-1637, indexed in Pubmed: 12042290.

10. Greer IA. Thrombophilia: implications for pregnancy outcome. Thromb Res. 2003; 109(2-3): 73-81, indexed in Pubmed: 12706634.

11. Kupferminc JM. Management of thrombophilia in women with PVC. Thromb Res. 2005; 115 Suppl 1: 46-50, indexed in Pubmed: 15790154.

12. Onderoglu L, Baykal C, Al RA, et al. High frequency of thrombophilic disorders in women with recurrent fetal miscarriage. Clin Exp Obstet Gynecol. 2006; 33(1): 50-54, indexed in Pubmed: 16761541.

13. Robertson $\mathrm{L}, \mathrm{Wu} \mathrm{O}$, Langhorne $\mathrm{P}$, et al. Thrombophilia in pregnancy: a systemic review. Br J Haematol. 2006; 132: 171-196.

14. Johnson CM, Mureebe L, Silver D. Hypercoagulable states: a review. Vasc Endovascular Surg. 2005; 39(2): 123-133, doi: 10.1177/1538574405039 00201, indexed in Pubmed: 15806273.

15. McNamee KM, Dawood F, Farquharson RG, et al. Thrombophilia and early pregnancy loss. Best Pract Res Clin Obstet Gynaecol. 2012; 26(1): 91-102, doi: 10.1016/j.bpobgyn.2011.10.002, indexed in Pubmed: 22079389.

16. Singer JB. Candidate gene association analysis. Methods Mol Biol. 2009; 573: 223-230, doi: 10.1007/978-1-60761-247-6_13, indexed in Pubmed: 19763931

17. Skrzypczak J, Rajewski M, Wirstlein P, et al. [Incidence of hereditary thrombophilia in women with pregnancy loss in multi-center studies in Poland]. Ginekol Pol. 2012; 83(5): 330-336, indexed in Pubmed: 22708328

18. Sergi C, Al Jishi T, Walker M. Factor V Leiden mutation in women with early recurrent pregnancy loss: a meta-analysis and systematic review of the causal association. Arch Gynecol Obstet. 2015; 291(3): 671-679, doi: 10.1007/s00404-014-3443-x, indexed in Pubmed: 25193429.

19. Baumann K, Beuter-Winkler $P$, Hackethal A, et al. Maternal factor $V$ Leiden and prothrombin mutations do not seem to contribute to the occurrence of two or more than two consecutive miscarriages in Caucasian patients. Am J Reprod Immunol. 2013; 70(6): 518-521, doi: 10.1111/aji.12144, indexed in Pubmed: 23795816.

20. Bałajewicz-Nowak M, Pityński K, Milewicz T. [The 1691 G > A (factor $\mathrm{V}$ Leiden) and $1328 \mathrm{~T}>\mathrm{C}$ V coagulation factor polymorphisms and recurrent miscarriages]. Ginekol Pol. 2015; 86(1): 46-52, indexed in Pubmed: 25775875.

21. Barlik M, Seremak-Mrozikiewicz A, Kraśnik W, et al. [The 20210G>A and $19911 \mathrm{~A}>\mathrm{G}$ polymorphisms of prothrombin gene and recurrent miscarriages]. Ginekol Pol. 2013; 84(10): 830-834, indexed in Pubmed: 24273903.

22. Gao H, Tao Fb. Prothrombin G20210A mutation is associated with recurrent pregnancy loss: a systematic review and meta-analysis update. Thromb Res. 2015; 135(2):339-346, doi: 10.1016/j.thromres.2014.12.001, indexed in Pubmed: 25528068.

23. Mrozikiewicz PM, Cascorbi I, Ziemer S, et al. Reduced procedural risk for coronary catheter interventions in carriers of the coagulation factor VII-GIn353 gene. J Am Coll Cardiol. 2000; 36(5): 1520-1525, indexed in Pubmed: 11079652.

24. Seremak-Mrozikiewicz A, Drews K, Kurzawińska G, et al. Związek polimorfizmu Arg353GIn czynnika VII krzepnięcia z poronieniami nawracającymi. Ginekol Pol. 2009; 80: 8-13.

25. Seremak-Mrozikiewicz A, Drews K, Kurzawinska G, et al. The significance of $1793 \mathrm{G}>$ A polymorphism in MTHFR gene in women with first trimester recurrent miscarriages. Neuro Endocrinol Lett. 2010; 31(5): 717-723, indexed in Pubmed: 21173738.

26. Robertson $\mathrm{L}, \mathrm{Wu} \mathrm{O}$, Langhorne $\mathrm{P}$, et al. Thrombophilia in pregnancy: a systemic review. Br J Haematol. 2006; 132: 171-196.

27. Frosst $\mathrm{P}, \mathrm{Blom} \mathrm{HJ}$, Milos $\mathrm{R}$, et al. A candidate genetic risk factor for vascular disease: a common mutation in methylenetetrahydrofolate reductase. Nat Genet. 1995; 10(1): 111-113, doi: 10.1038/ng0595-111, indexed in Pubmed: 7647779.

28. Hanson NQ, Aras O, Yang F, et al. C677T and A1298C polymorphisms of the methylenetetrahydrofolate reductase gene: incidence and effect of combined genotypes on plasma fasting and post-methionine load homocysteine in vascular disease. Clin Chem. 2001; 47(4): 661-666, indexed in Pubmed: 11274015.

29. Rady PL, Szucs S, Grady J, et al. Genetic polymorphisms of methylenetetrahydrofolate reductase (MTHFR) and methionine synthase reductase (MTRR) in ethnic populations in Texas; a report of a novel MTHFR polymorphic site, G1793A. Am J Med Genet. 2002; 107(2): 162-168, indexed in Pubmed: 11807892.

30. Bertina RM, Koeleman BP, Koster T, et al. Mutation in blood coagulation factor $V$ associated with resistance to activated protein C. Nature. 1994; 369(6475): 64-67, doi: 10.1038/369064a0, indexed in Pubmed: 8164741.

31. Faisel F, Romppanen EL, Hiltunen M, et al. Susceptibility to pre-eclampsia in Finnish women is associated with R485K polymorphism in the factor $\mathrm{V}$ gene, not with Leiden mutation. Eur J Hum Genet. 2004; 12(3): 187-191, doi: 10.1038/sj.ejhg.5201124, indexed in Pubmed: 14673478.

32. Poort SR, Rosendaal FR, Reitsma PH, et al. A common genetic variation in the 3-prime-untranslated region of the prothrombin gene is associated with elevated plasma prothrombin levels and an increase in venous thrombosis. Blood. 1996; 88: 3698-3703.

33. Green $F$, Kelleher $C$, Wilkes $H$, et al. A common genetic polymorphism associated with lower coagulation factor VII levels in healthy individuals. Arterioscler Thromb. 1991; 11(3): 540-546, indexed in Pubmed: 1709359. 